\title{
Determine Operating Reactor to Use for the 2016 PCI Level 1 Milestone
}

Kevin Clarno, Oak Ridge National Laboratory January 30, 2016
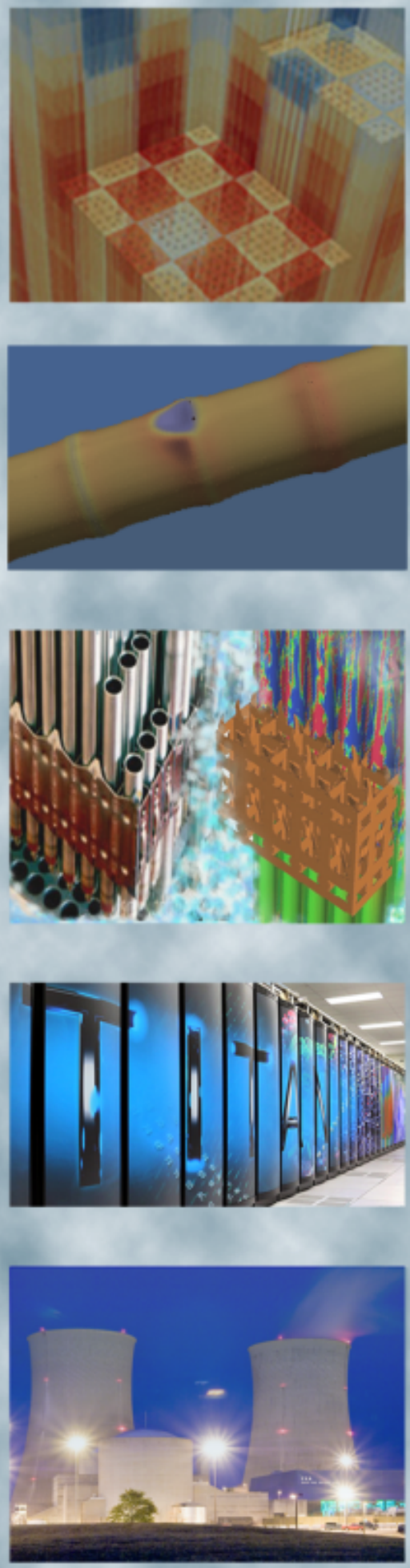
REVISION LOG

\begin{tabular}{|c|c|c|l|}
\hline Revision & Date & Affected Pages & \multicolumn{1}{c|}{ Revision Description } \\
\hline 0 & & All & Initial Release \\
\hline & & & \\
\hline & & & \\
\hline & & & \\
\hline
\end{tabular}

\section{Document pages that are:}

Export Controlled

IP/Proprietary/NDA Controlled

Sensitive Controlled

\section{Requested Distribution:}

To: Jess Gehin

Copy: Brian Writh, Jess Gehin, Andrew Godfrey, Scott Palmtag, Ben Collins, Shane Stimpson, Roger Pawlowski, Jeff Powers, Richard Williamson 


\section{EXECUTIVE SUMMARY}

The Consortium for Advanced Simulation of Light Water Reactors (LWRs) (CASL) Level 1 milestone to "Assess the analysis capability for core-wide [pressurized water reactor] PWR PelletClad Interaction (PCI) screening and demonstrate detailed 3-D analysis on selected sub-region" (L1:CASL.P13.03) requires a particular type of nuclear power plant for the assessment. This report documents the operating reactor and cycles chosen for this assessment in completion of the physics integration (PHI) milestone to "Determine Operating Reactor to use for PCI L1 Milestone" (L3:PHI.CMD.P12.02). Watts Bar Unit 1 experienced (at least) one fuel rod failure in each of cycles 6 and 7, and at least one was deemed to be duty related rather than being primarily related to a manufacturing defect or grid effects. This brief report documents that the data required to model cycles 1-12 of Watts Bar Unit 1 using VERA-CS contains sufficient data to model the PHI portion of the PCI challenge problem. A list of additional data needs is also provided that will be important for verification and validation of the BISON results. 


\section{CONTENTS}

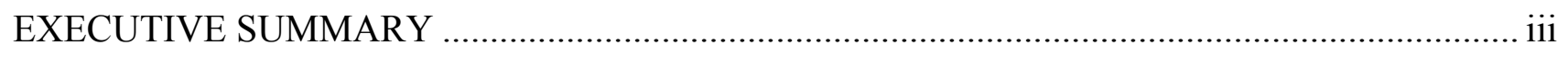

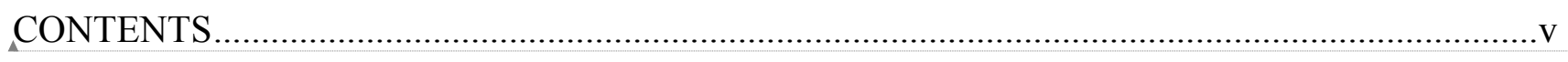

TABLES ............................................................................Error! Bookmark not defined.

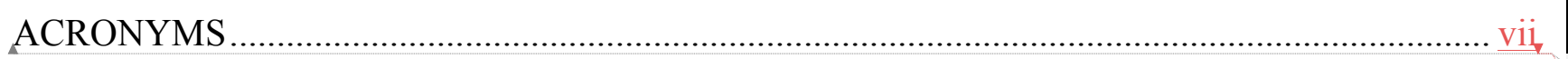




\section{ACRONYMS}

CASL Consortium for Advanced Simulation of Light Water Reactors

CIPS

$\mathrm{CP}$

CRUD-induced power shift

CPI

challenge problem

CRUD

challenge problem integrator

DOE

corrosion-related unidentified deposits or Chalk River unidentified deposits

DOE NE

US Department of Energy

FA

US Department of Energy Office of Nuclear Energy

FMC

$\mathrm{Gd}_{2} \mathrm{O}_{3}$

IFBA

INL

IP

focus area

Fuel, Materials and Chemistry

gadolinium (III) oxide

integral fuel burnable absorber

Idaho National Laboratory

LWR

MOX

NPP

intellectual property

light water reactor

mixed oxide

nuclear power plant

ORNL

PCI

Oak Ridge National Laboratory

PCMI

PHI

pellet-cladding interaction

pellet-cladding mechanical interaction

physics integration

PIE

PWR

post-irradiation examination

TVA

pressurized water reactor

UIUC

Tennessee Valley Authority

VERA

University of Illinois at Urbana-Champaign

WEC

Virtual Environment for Reactor Applications

Westinghouse Electric Company 



\section{BACKGROUND}

In FY15, the Fuel, Materials and Chemistry (FMC) Focus Area (FA) completed a Level 2 milestone to model the missing pellet surface (MPS) fuel failures in the Braidwood Nuclear Power Plant (NPP). In FY16, the Pellet-Cladding Interaction (PCI) Challenge Problem (CP) will be extended to provide a capability for core-wide pressurized water reactor (PWR) PCI screening and demonstration of a detailed 3-D analysis on the selected subregion, which culminates in a Level 1 milestone. For this milestone, the Physics Integration (PHI) FA component will include developing the core-wide capability to model the nominal operation of the NPP using VERA-CS and the corresponding BISON fuel performance predictions of a suite of PCI indicators for multiple cycles of operations. Then FMC will use the results to screen for potential PCI failures and perform detailed 3-D analyses on selected subregions. Finally, the PCI Challenge Problem Integrator (CPI) will assess the capability of the VERA toolset. Identification of the particular cycles of a particular NPP will define the scope and relevance of the final results.

The Braidwood NPP was the original choice for the analysis because it had experienced known fuel failures that were modeled with BISON in FY15, and because of plans with Exelon to acquire the data for full core analysis in FY16. Since the choice of the reactor would define many aspects of the Level 1 milestone and the work required of FMC and PHI, a Level 3 milestone was established to identify the particular cycles of a particular NPP to be used in the Level 1 milestone.

\section{REQUIREMENTS}

The choice of the NPP is significant for a host of reasons. If there are no fuel failures, or all failures are due to manufacturing defects, then the screening tool should provide relatively uninteresting results; therefore, an NPP that experienced fuel failures due to a traditional pellet-clad mechanical interaction (PCMI) is needed to assess the quality of the screening tool. Establishing the inputs for VERA-CS and BISON requires loading patterns and operational history from the utility, as well as fuel specifications from the fuel vendors. The utilities for some NPPs have these data readily available for every cycle, while other utilities must search microfiche from the 1970s and process the data accordingly. NPPs with fuel from multiple vendors may have additional challenges in sharing their data due to multiple nondisclosure agreements restricting the distribution of data between vendors.

The cycles evaluated for the chosen NPP define the capability required of BISON and VERA-CS. BISON may need to include the capability for modeling the unique differences between the standard $\mathrm{UO}_{2}$ fuel rods and those containing mixed oxide (MOX), gadolinium-oxide $\left(\mathrm{Gd}_{2} \mathrm{O}_{3}\right)$, and integral fuel burnable absorber (IFBA). Similarly, if the plant includes fixed in-core detectors or others features, VERA-CS would need to have the capability to model them accordingly. Also, some plants have experienced operational transients in a particular cycle that affect that fuel and even those in subsequent cycles. For example, in cycle 7 of Watts Bar Unit 1, there was a significant axial power anomaly due to CRUD (Chalk River unidentified deposits); this CRUD-induced power Shift (CIPS) substantially altered the axial power distribution in cycles 7 and the axial burnup distribution in the fuel that remained in core, also altered the power distribution in cycle 8. Neglecting the CRUD growth would dramatically change the PCI indicators predicted by VERA.

To assess the quality of the simulation capability, each portion of the problem must be validated. Each NPP performs a suite of startup testing and monitoring of reactor operation during each cycle. The accuracy of the VERA-CS results can be demonstrated through comparisons with isothermal reactivity coefficients, control rod bank worths, boron letdown curves, and flux maps. The accuracy of the BISON results can be demonstrated through comparison with post-irradiation examination 
(PIE) of any fuel rods examined. Therefore, it is important to acquire this data for the cycles of interest, as well as those preceding it.

\section{DECISION}

During the Joint Industry Council/Science Council meeting in October 2015, Joe Rashid of Anatech mentioned that there were fuel failures in Watts Bar Unit 1. Further investigation at the PCI meeting at Anatech in early December revealed that a single fuel pin failed in each of cycles 6 and 7 and that at least one of those failures was duty related. Based on the work performed for two major milestones for VERA-CS in FY15, it was clear that VERA-CS could accurately model cycles 1-12 of Watts Bar Unit 1 and could even account for the CIPS that occurred in cycle 7. Therefore, it was decided that cycles 6-7 would be used for the level 1 PCI milestone in FY16.

Collaboration with Exelon related to the MPS failures is continuing within FMC, as well as PHI, through the Applied Research Institute of the University of Illinois at Urbana-Champaign. While it is currently focused on the Byron NPP, if the Braidwood data become available in the coming year and the computer resources are available (through CASL or at University of Illinois at UrbanaChampaign [UIUC]), additional assessments of the PCI predictive capability can be performed.

\section{ADDITIONAL NEEDS}

During this process, several additional needs were defined. The capability to provide the integrated screening tool is currently in development within PHI as part of existing milestones. Westinghouse Electric Company (WEC) and the Tennessee Valley Authority (TVA) were closely associated with the FY15 level 1 milestones for VERA-CS and have independently verified the input and analysis. However, an independent review of the BISON inputs with all default parameters and models defined would help to confirm that the fuel is being modeled correctly. If WEC does not disclose all of the differences in the BISON input and models due to the highly proprietary nature of nuclear fuel, it would be beneficial to have them quantify the errors in the PCI indicators due to differences in models and inputs.

CASL has not received official confirmation from TVA regarding the fuel failures. Before the assessment phase, it will be necessary to have identified the particular pins that failed in each cycle and the relevant information as to why the pins failed. If the PIE data were accessible for each of the failed fuel rods, it would provide very useful validation.

Any other fuel rods that were placed in Watts Bar Unit 1 cycles 1-12 that also have PIE data would provide tremendous benefit for validation of BISON in both 2-D and 3-D modes. 